\title{
A Preliminary Study on Adaptability of College Students in the New Era and Its Cultivating Model
}

\author{
Feng Xiao', Wei Hua², Juanmei Han¹, Ru Yan ${ }^{1}$ \\ ${ }^{1}$ Guangdong University of Foreign Studies, Guangzhou, China \\ ${ }^{2}$ Nanjing Tech University, Nanjing, China \\ Email: xiaofeng@gdufs.edu.cn
}

How to cite this paper: Xiao, F., Hua, W., Han, J. M., \& Yan, R. (2020). A Preliminary Study on Adaptability of College Students in the New Era and Its Cultivating Model. Psychology, 11, 2055-2065.

https://doi.org/10.4236/psych.2020.1112128

Received: November 2, 2020

Accepted: December 27, 2020

Published: December 30, 2020

Copyright $\odot 2020$ by author(s) and Scientific Research Publishing Inc. This work is licensed under the Creative Commons Attribution International License (CC BY 4.0).

http://creativecommons.org/licenses/by/4.0/

\begin{abstract}
As part of the most important components of college students' psychological quality structure, their adaptability cultivation should not be ignored. This paper starts from the significance of adaptability for improving psychological quality education model and puts forward specific stage-and-content based strategies for the cultivating of college students' adaptive ability through discussion about psychological mechanism of adaptability cultivation, that is, the first year is stage one, the second and third years are stage two and the fourth year is stage three. And then it further proposes cultivating objectives, key content and strategies in different stages.
\end{abstract}

\section{Keywords}

Adaptability, Cultivating Model, College Students

\section{Research Background}

With the deepening of theoretical research and practical application of psychological quality education, the model, approach, strategy and evaluation of psychological quality education have gradually become the difficulties and hot issues of psychological quality education. Both theoretical research and practical application are in urgent need of further improvement, but current academic discussion on the model of psychological quality education is not enough.

Zhou Dongfang believes that psychological quality is the core of people's quality structure. Modern society requires comprehensive and systematic training of people's quality, especially psychological quality (Zhou, 2001). Wang Ping has concluded on the basis of practice that the education model of college stu- 
dents' psychological quality includes "three three six six one integration", that is, to establish three organizations, form a three-dimensional consulting team, combine six factors, achieve six goals, and finally achieve one integration (Wang, 2001). Zhao Hong has discussed the principle and specific content of establishing objective model of normal college students' psychological quality education, operation mode and evaluation mode of psychological quality education, and put forward seven operation modes (Zhao, 2006). Li Yushan has introduced an open and dynamic school psychological education model which was implemented in the local area with the developmental psychological education as the orientation, the psychological education research as the guide, the psychological education curriculum as the core, the students' self-education as the emphasis, and the scientific evaluation as the means (Li, 2002b). Hu Xiaomei believes that in the new media environment, we should build a student-centered education model, implement mental health education with the help of information education platform of psychological quality, and effectively enhance students' comprehensive psychological quality $(\mathrm{Hu}, 2017)$. The author believes that there are three problems in existing research. First, it is more descriptive and less theoretical; second, there are more macro studies and less micro-analysis; third, with more attention to normal university students and those in compulsory education stage, there's a lack of attention and analysis of the wider group of college students in the new era. Through the important role of adaptability in the psychological quality of college students, and discussion on how to cultivate such an adaptability, this paper intends to start from the adaptability of college students to deepen the research on the cultivating model of college students' psychological quality.

\section{Importance of Cultivation of College Students' Adaptability and Its Psychological Mechanism}

\section{1) Importance of cultivation of college students' adaptability}

"The Comprehensive Dictionary of Psychology" edited by Zhu Zhixian defines "adaptation" as "a term derived from biology, which is used to indicate those physical and behavioral changes that can increase the chances of an organism's survival. In psychology, it is used to express the response to environmental changes, such as adaptation to changes in light and changes in human social behavior. Piaget thinks that from the biological point of view, the essence of wisdom is adaptation, which can be either a process or a state. The organism is in constant motion and changes to achieve balance with the environment, and the process can be summarized as two complementary parts: assimilation and accommodation, and the adaptation state is the result of relative balance between the two parts. This kind of balance is not absolutely static, and one level of balance will lead to the beginning of another level of balance movement; if the body and the environment are out of balance, it is necessary to change behavior to rebuild balance. The dynamic process of balance-imbalance-balance is adapta- 
tion, which is the essence and reason of children's intellectual development" (Zhu, 1989). Based on this definition, adaptation in psychology mainly includes three aspects: physiology, psychology and society, which cover almost everything in psychological life. Piaget even regards adaptation as the basic motive force of individual development.

Since adaptability is so important for personal development, then what kind of role does it play in the structure of college students' psychological quality? With the analysis of students' psychological quality structure, discussion of the meaning and essence of psychological quality as the theoretical starting point, and practical basis of the open questionnaire of educational psychology experts, college and middle school teachers and students, Zhang Dajun et al. (Zhang et al., 2000) has summarized the students' psychological quality into three basic dimensions: cognition, personality and adaptability, and analyzed the relationship among the three dimensions from the structure-function perspective. Cognition elements are shown in the reflection of human beings to objective things, they directly participate in the specific practice of cognition of objective things and are the basic component of psychological quality. Personality elements are shown in the treatment of human beings to objective things. Although they do not directly participate in the specific practice of cognition of objective things, they motivate and adjust cognition practice, and are the driving force of psychological quality. The so-called function of psychological quality is the adaptability based on certain elements of psychological characteristics (cognition quality and personality quality), through the interaction with the external environment, it selects, adapts to and changes the environment, so as to make itself coordinate with the environment, that is, the adaptability in the structure of psychological quality (Zhang, 2004). On this basis, Wang Tao regards adaptability as one of the three major qualities in the psychological quality structure of people at college age through a large-scale questionnaire survey (Wang, 2002). The author believes that these studies fully show the position and role of adaptability in psychological quality, that is, adaptability is one of the most important components in the structure of college students' psychological quality.

\section{2) Psychological mechanism of college students' adaptability cultivation}

Theoretically speaking, there are two basic ways for an individual to adapt to the environment: one is to change oneself to adapt to the environment; the other is to stick to one's own principles to change the environment. To change the environment means to make the political, economic, cultural and educational activities of the society more conducive to one's adaptation and development; to change the individual means that the individual, facing the new living environment, adjusts himself mentally and physically and learns to adapt. In real life, we put more emphasis on adapting to the surrounding environment by adjusting oneself mentally and physically, so this paper will look at the psychological mechanism of adaptation from this perspective. Jia Xiaobo believes that the mechanism of psychological adaptation is a dynamic process composed of cogni- 
tion adjusting, attitude changing, behavior selecting and others, and the related psychological quality is the key to the cultivating of psychological adaptability (Jia, 2001). The cultivating of college students' adaptability should be targeted based on this process. When facing a new environment, college students often have resistance and refuse to change, which leads to maladjustment. Then they begin to evaluate this situation, see what changes have taken place, what impact these changes will have on them and so on. This kind of internal evaluation promotes changes in their self-experience, make them change their goals or change themselves to adapt to new changes, so they can readapt. In this process, the adaptability of college students has been improved. According to this dynamic process of psychological adaptation, this paper puts forward the specific stage-and-content based strategies for the cultivating of college students' adaptive ability.

\section{The Cultivating Model of College Students' Adaptability}

There is a close relationship between mental health and psychological quality. A person can be mentally healthy only if he has good psychological quality. Therefore, mental health education must start with developing sound psychological quality. Adaptability is one of the most important parts of psychological quality structure. How to cultivate students' adaptability is an important part of mental health education and psychological quality cultivation. The author will go further into how to cultivate the college students' adaptability from objectives, content and methods.

1) The cultivating objectives and specific content of college students' adaptability

Different scholars have different understanding of the goal of school psychological quality education. Li Jun believes that the goal of psychological health education should include three levels: specific goal, intermediate goal and ultimate goal (Li, 2002a). The specific goal is to cultivate various specific psychological health factors; the intermediate goal is to coordinate the development of various psychological health factors and promote the development and improvement of individual mental health; the ultimate goal is to promote the comprehensive, coordinated and healthy development of individuals on the basis of good mental health education. Zhang Dajun and others think that the goal system of school psychological quality education includes stratified goal, age groupbased goal and classified goal. According to the different functions of psychological quality education, the stratified goal system divides the objectives of psychological quality education into three levels: guiding students to adapt actively and maintaining their physical and mental health; promoting active development to form sound psychological quality; cultivating creativity and improving creative psychological quality. According to the different degrees of physical and mental development of students of different ages, the age group-based goal system plans individual cultivating objectives. The classified goal system, based on the content 
of psychological quality education, divides the objectives of psychological quality education into three major aspects: cognition, personality and adaptability, and each aspect includes its own content. This paper, combined with Zhang Dajun and others' research on the goal system of psychological quality education, holds that the goal of college students' adaptability cultivation in the new era should fully implement the three levels of stratified goal. We should not only pay attention to students' various adaptations, but also promote their all-round development and improve their creativity. The cultivating goals of college students' adaptability should also be varied in different age groups.

Ma Jianqing's research shows that the first-year college students have problems with adaptation as freshmen, also with study, interest in major, and classmates relations as well; in the second grade, the problems are interpersonal relations, study and career, love and relationships and so on; in the third grade, the main problem is love and relationship, and there are also problems of self-development and ability cultivating, interpersonal communication and others; and in the fourth grade, choice of career is the major problem, and there are also problems of relationship, future development, ability cultivating and so on $(\mathrm{Ma}$, 1998). This shows that there are differences in adaptation problems faced by college students in four years. If combined with the idea of Zhang Dajun and others that the adaptability is divided into study adaptation, life adaptation, physiological adaptation, occupational adaptation, social environment adaptation, interpersonal environment adaptation and emergency situation adaptation. The author thinks that the four years of university can be classified as three stages: the first stage is grade one, the second stage is the grade two and three, and the third stage is grade four. In each stage, the goal of adaptation should be different because the focus of each stage is different. The first stage focuses on life adaptation, social environment adaptation (this mainly refers to school and school surroundings at this stage), study adaptation and emergency situation adaptation; the second stage focuses on interpersonal environment adaptation and study adaptation; the third stage focuses on occupational adaptation and social environment adaptation (in fact, there are different degrees of physiological adaptation problems at each stage, but the author mainly discusses psychological adaptation, not physiological adaptation and others).

\section{2) Ways to cultivate the adaptability of college students}

Combined with the above-mentioned three stages of different content and objectives of college students' adaptation, the author believes that the cultivating of college students' adaptive ability should also be carried out according to different stages and content for specific cultivating and education.

a) Stage one (first year of college)

The main problems faced by freshmen are life adaptation, social environment adaptation and stress adaptation. When freshmen are in a new environment, not only has the natural environment such as climate and temperature changed greatly, but also the people they meet are strange. What's more, their past life 
style may change completely. When a person moves from one cultural area or cultural circle to another, he will face the challenge from unfamiliar cultural environment, which will produce the need for adaptation and coping, and stress reaction as well (Li, 2004). So the most important problem faced by freshmen is to adapt to life, social environment and stressful situation. In order to cultivate this adaptability of college freshmen, on the one hand, it is necessary to do well in the connection of psychological quality education, that is, to do well in the connection of psychological quality education between high school and college. This is a hot and difficult issue in psychological quality education at present. It is emphasized that students' ability to adapt to new environment and to live independently should be cultivated in high school. On the other hand, we should do a good job in the admission reception and orientation of freshmen. Many adaptation problems of college students, including some psychological disorders, are related to the imperfections of traditional admission reception and orientation. The traditional admission reception and orientation are more like a ritual or routine ceremony, in which the senior students help the freshmen carry luggage, show them the dormitory, and tell them about which cafeteria is cheaper and better; the counselor teachers give some speeches on ideological education and explain the school rules and regulations in detail. These are not so much to help freshmen adapt to the new life and new environment as they are to create pressure and increase difficulties for freshmen. As a result, we should not only pay attention to the above-mentioned activities, but also make full use of the good opportunity of freshmen entering college. Through a lot of theoretical research and practical research in primary and middle schools, Zhang Dajun and others proposed a home-school cooperation model for psychological quality education, which validates the important supporting role of family and community in the cultivating of psychological quality. However, how to improve the psychological quality of college students through home-school cooperation has always been a problem. The author believes that, on the one hand, most parents will send their children to school to register, so we should use this opportunity to let the parents know about relevant knowledge, and reach a consensus with them on strengthening the adaptability of freshmen, establish the home-school cooperation to fully play the role of families in improving the psychological quality of college students. On the other hand, the admission orientation should be reformed, not limited to the dogmatic content, there should be some more to help freshmen adapt to the new environment faster and better, such as introducing the school history, development, talent cultivating and discipline advantages, as well as study, living environment and surroundings of the school and so on. This not only helps freshmen better adapt to life and environment, but also lays the foundation for the development of learning adaptability at the second stage and strengthens the cohesion of psychological quality cultivating.

b) Stage two (second and third years of college)

As time goes by, by the time of the second and third years, most of the stu- 
dents have adapted to the environment and life of college, and have begun the major part of college life-study and communication. However, Wang Ling believes that "the relationship between students is limited to the interaction in life and some exchanges in study, and lacks in-depth communication, so it's very common that college students feel lonely, they lack necessary interpersonal communication and support in the face of fierce competition, forming the external environment of psychological problems" (Wang, 1995). If college students can't regard their own shortcomings correctly, or fail to deal with all kinds of setbacks and inner conflicts properly, or they are not good at self-regulation with emotions, all these will affect their study and life. In the practice of college students' psychological guidance, the author realized that it is not enough to rely on psychological counselling clinic, because the nature of psychological counselling determines that it is just "sitting and waiting for others to consult". At present, the number of students who take the initiative to go to the schools' psychological counseling center for consultation is generally only $1 \%-2 \%$, and there is still a big gap between this and about $20 \%$ of students who need help with potential psychological problems. This indicates that college students do not study and communicate well, and they need professional guidance; and once there are communication or study problems, they do not know how to ask for help. Although almost every college has established a mental health education center or similar institutions, they have not played their due role due to various reasons.

The behavior of seeking medical help when people feel sick or have symptoms is called health care seeking. A survey shows that when Chinese people have psychological distress, the channels of psychological adjustment are self-regulation, close friends, family, colleagues, and social consultancies. In other words, Chinese people are not willing to seek help from specialized mental health institutions when facing psychological distress ( $\mathrm{Li} \& \mathrm{Gao}, 2007$ ). Therefore, it is necessary to take other forms to help college students adapt to study and communication. Zhang Dajun thinks that "school mental health education is a systematic project, which needs the collaborative effect of the whole education system, and at the same time, the unique role of each element of education should be fully utilized. We should give full play to the main role of students, the leading role of school education, supporting role of family and strengthening role of community in mental health education" (Zhang, 2002). However, college students are a special group and college campus is a special campus. The departments are relatively loose, so it is difficult for the whole school to play the leading role. Therefore, the author believes that the coordination and integration model should be established, which is student center - department lead - class support - dormitory strengthen, so that the students, departments, classes and dormitories can perform effectively. The research conducted by Qu Zhiyong, Zou Hong and Wang Yingchun shows that the class environment can be classified into three types: united and progressive, general, and problematic. In terms of school liking, interest in study, academic efficacy and self-confidence, the students in the 
united and progressive classes score significantly higher than those in the general and problematic classes. Class environment has a significant positive predictive effect on students' school liking and academic behavior, a significant negative predictive effect on school avoidance, and an indirect effect on academic behavior greater than direct effect (Qu, Zou et al. 2004). Therefore, the establishment of a consistent college culture, department culture, class culture and dormitory culture that is conducive to the formation and development of students' healthy psychological quality can give full play to the beneficial effects of college, department, class and dormitory.

We should change the traditional one-on-one outpatient consultation to an invitation-based group consultation. The so-called invitation-based model requires a school to make full use of school personnel, material conditions, rules and regulations, education policies, various educational plans and educational processes, so as to make the school the most attractive place for everyone. Here, everyone feels welcomed, respected, accepted and appreciated. The school encourages teachers and students to realize their potentials and respect the unique values of themselves and others. The school's psychological guidance to students is not passive or rigid, but is an educational activity that students are willing to accept (Purkey \& Schmidt, 1990). We should change the passive consultation method, instead, actively invite students with common problems, their roommates, members of their small groups, and classmates to discuss the study adaptation and interpersonal issues, so as to solve the common problems; or set a certain situation or psychological games for these members to participate in, so that they can experience, reflect, develop quality, and improve their psychological health.

Finally, a sound social support system must be established. Social support refers to the spiritual and material support and help that one can generally feel from family members, relatives and friends, organizations and other social groups. Most of the existing studies show that good social support is beneficial to physical and mental health, while the existence of bad social relations harms physical and mental health. On the one hand, social support provides protection to the individual under stress, that is, the buffering effect. On the other hand, it is of great significance to maintain the general good emotional experience. Li Qiang believes that the social support mechanism that China will reconstruct in the future should include the following structural elements: first, the government-led social security system, which is the main channel to provide social support for vulnerable groups and reduce the impact of life crisis on their family life and personal psychology; second, social support provided by non-governmental organizations (including specialized mental health institutions, workplaces, social organizations such as Labor union, Communist Youth League and women's Federation, social charity organizations, community service organizations and religious organizations); third, personal support based on blood relationship, geographical relationship, professional relationship, human- 
ism and philanthropy. But the social support system discussed here is mainly the social support system within the school. This support system should give full play to the various elements of school education and provide a full range of services to students. First, schools should provide policy and economic support to ensure the smooth operation of the system; second, it should give full play to the professional and publicity advantages of the school's mental health education center, provide students with professional help and expand the influence of publicity, and correct people's misconceptions on psychological counseling; third, we should fully mobilize managerial teachers and counselor teachers in charge of student affairs in each department to change their thoughts and actively cooperate to provide students with ideological guarantee; fourth, we also give full play to the mental health associations in schools. Studies have shown that "on the basis of traditional mental health interventions, school mental health associations can popularize psychological knowledge among students on a larger scale, carry out mental practice service activities, and form a good mental education and mental health care environment" (Yang, Yan et al., 2000).

c) Stage three (fourth year of college)

At this stage, college life is coming to an end. Most students will leave schools and work, so the issue is mainly about adapting to the future career and social environment.

This problem has long been ignored by colleges. Most colleges believe that once a student graduates from the school, the school has completed its job of teaching, and it's a matter of student personal development to adapt to the social environment and future career. However, with the progress of higher education reform, more and more colleges realize that it is necessary and obligatory to provide employment and vocational education for college students. On this issue, there are currently the following misunderstandings: formalism in the purpose and content of education; utilitarianism in educational approaches and methods-taking employment and vocational education as a short-term training; simplification of educational subject; passivity and non-transparency in information exchange. These caused various career mistakes made by college graduates. Therefore, the author believes that it is necessary to explore an effective employment and vocational education model.

First of all, colleges should change the way of thinking, strictly implement the requirements of the Ministry of Education, and include employment and vocational education as a compulsory course for cultivating students' quality, rather than just as a part of the vanity project or formalism. Secondly, the content of employment and vocational education should be further improved. We should have comprehensive education on the career choice, employment and students' self-positioning, and the following contradictions should be handled correctly: the contradiction between social needs and personal professional interest, between personal professional abilities and intellectual expertise, between professional ideal and employment reality, between immediate interests and future 
development prospects, between career stability and preparation for career mobility and so on. Thirdly, we should combine the whole course and short-term feature of employment and vocational education, and focus on the subject role and practice of students, so that students can improve their comprehensive quality in daily social practice.

\section{Conclusion}

With the pandemic, the psychological problems of college students present a high incidence. As one of the most important components of college students' psychological quality structure, the cultivating of adaptability can't be ignored. Starting from the cultivating of adaptability, the author focuses on the important position of adaptability in the psychological quality of college students, the psychological mechanism of adaptation and its enlightenment on the cultivating of college students' adaptability, analyzed and discussed in detail the cultivating objectives, specific content and methods according to different stages and content, with the aim to further deepen the theoretical and practical research on the psychological quality cultivation of college students. Due to the limitation of space, the author did not discuss the other parts of college students' psychological quality structure and their interaction with adaptability, which is still worthy of attention. In short, the author expects to further enrich the theory and practice of college students' psychological quality education by exploring the adaptability of college students.

\section{Conflicts of Interest}

The authors declare no conflicts of interest regarding the publication of this paper.

\section{References}

Hu, X. M. (2017). An Analysis on the Innovation of College Students' Psychological Quality Education Model in the New Media Environment. Culture Journal, No. 4, 114-116.

Jia, X. B. (2001). The Nature and Mechanism of Mental Adaptation. Journal of Tianjin Normal University (Social Science), No. 1, 19-23.

Li, J. (2002a). On the Establishment of Mental Health Education System of College Students. Journal of Jinan University (Social Science), No. 4, 86-88.

Li, Q. (2004). Causes and Intervention of Mental Health Problems in China during the Period of Social Transformation. Theory and Modernization, No. 1, 31-35.

Li, Q., \& Gao, W. J. (2007). Coping Method of Chinese People's Psychological Distress and Its Social and Cultural Roots. Theory and Modernization, No. 5, 108-114.

Li, Y. S. (2002b). A Preliminary Study on the Model of School Psychological Quality Education. Education Exploration, No. 2, 96-98.

Ma, J. Q. (1998). Developmental Counseling: The Basic Model of School Psychological Counseling. Contemporary Young Research, No. 1, 7-11.

Purkey, W. W., \& Schmidt, J. J. (1990). Invitational Learning for Counseling and Devel- 
opment. Ann Arbor, MI: The University of Michigan, ERIC/CAPS.

Qu, Z. Y., Zou, H. et al. (2004). Class Environment: Types and Its Prediction on Students' School Adjustment. Psychological Science, No. 1, 207-211.

Wang, L. (1995). College Students' Psychological Problems and Countermeasures. Forward Position, No. 5, 41-42.

Wang, P. (2001). Research on College Students' Psychological Quality and Education Model. China Higher Education Research, No. 10, 55-56.

Wang, T. (2002). Research on the Structure of College Students' Psychological Quality and Its Development Characteristics. Master's Thesis, Chongqing: Southwest China Normal University, Institute of Education Sciences.

Yang, Y. R., Yan, L. L. et al. (2000). The Influence of Mental Health Clubs on College Students' Psychological Quality. Chinese Journal of School Doctor, No. 1, 64-65.

Zhang, D. J. (2002). Strengthen Mental Health Education in Schools and Foster Students' Psychological Quality. Journal of Hebei Normal University (Educational Science), No. 1, 17-23.

Zhang, D. J. (2004). An Introduction to School Psychological Quality Education. Chongqing: Southwest China Normal University Press.

Zhang, D. J. et al. (2000). Problems on Research of Students' Mental Quality. Journal of Southwest China Normal University (Humanities and Social Sciences), No. 3, 56-62.

Zhao, H. (2006). Model of Psychological Quality Education in Teachers' College. Journal of Northeast Normal University (Philosophy and Social Sciences), No. 6, 184-188.

Zhou, D. F. (2001). Strengthening Psychological Quality Education is the Key Task of Constructing Quality Education Model. Education Exploration, No. 2, 63.

Zhu, Z. X. (1989). The Comprehensive Dictionary of Psychology. Beijing: Beijing Normal University Publishing House. 